\title{
Cytogenetic Characterization of Brazil Origin Marajoara Horses
}

\author{
Tiago Marafiga Degrandi ${ }^{1}$, José Ribamar Felipe Marques ${ }^{2}$, Maria Rosa Travassos \\ Da Costa ${ }^{2}$, Lucia Vinade ${ }^{1}$, Ricardo José Gunski ${ }^{1}$ and Analía Del Valle Garnero ${ }^{1}$ \\ ${ }^{1}$ Federal University of Pampa (UNIPAMPA), Av. Antônio Trilha, São Gabriel, RS, Brazil \\ ${ }^{2}$ Brazilian Agricultural Research Corporation (EMBRAPA) - Eastern Amazon Unit, Trav. Dr. Enéas \\ Pinheiro s/no Caixa Postal, Belém, PA, Brazil
}

Correspondence should be addressed to: Tiago Marafiga Degrandi; tdegrandi@hotmail.com

Received 16 April 2013; Accepted 21 May 2013; Published 28 August 2013

Academic Editor: Arunachalam Arangasamy

Copyright (C 2013 Tiago Marafiga Degrandi, José Ribamar Felipe Marques, Maria Rosa Travassos Da Costa, Lucia Vinade, Ricardo José Gunski and Analía Del Valle Garnero. Distributed under Creative Commons CC-BY 3.0

\begin{abstract}
The Marajoara breed of horses is an important animal group genetically conserved on the island of Marajó, State of Pará, Brazil. This breed exhibits very specific characteristics, but has suffered decharacterization as a result of crossing with other naturalized breeds in Brazil. For this reason, Marajoara horses have been introduced into the National Genetic Conservation Program, which includes both ex situ and in situ preservation, and also involves establishing productive, reproductive, genetic and phenotypic parameters for Marajoara horses. Therefore, the aim of this study was the cytogenetic characterization of the Marajoara horses. In order to do it we used the peripheral blood lymphocyte cultures of eight specimens, evaluated in terms of diploid number, morphology, chromosome biometry and C- and NOR-banding patterns. All specimens evaluated had $2 \mathrm{n}=64$ chromosomes and karyotype consisting of submetacentric pairs ( 1 to 11,13 and X), a metacentric pair (12) and telocentric pairs (14 to 31 and Y). Cbanding was observed marking centromeric of all complement and interstitial marking feature for $\mathrm{X}$ and $\mathrm{Y}$ chromosome almost completely heterochromatic. Nucleolus organizer regions were observed in pair 1 in the telomeric region, 28 in interstitial short arm and in 31 near the centromere. The cytogenetic characterization of these animals is the first step in the process of selecting animals for reproduction by establishing the diploid number as a basis for subsequent actions in the genetic conservation program.
\end{abstract}

Keywords: Chromosome, conservation, extinction, breed.

\section{Introduction}

In Brazil, horses play an extremely important role in agricultural activities involving the management of cattle, buffalo and sheep over extensive agricultural systems by Marques et al., (2003). Since they were introduced in Brazil, these horses have been selected for this kind of work over many generations and have developed in localized characteristic genetic groups, also referred to as

Cite this Article as: Tiago Marafiga Degrandi, José Ribamar Felipe Marques, Maria Rosa Travassos Da Costa, Lucia Vinade, Ricardo José Gunski and Analía Del Valle Garnero (2013), "Cytogenetic Characterization of Brazil Origin Marajoara Horses," International Journal of Veterinary Medicine: Research \& Reports, Vol. 2013 (2013), Article ID 829370, DOI: 10.5171/2013.829370 
naturalized Brazilian breeds by Mariante and Egito, (2002). In 1993, was set up a national program for conservation of genetic resources involving various animals of different genetic groups, including breeds of the horses Lavradeiro in the State of Roraima, Pantaneiro in Mato Grosso do Sul, Campeiro in Santa Catarina and Marajoara on the island of Marajó, Pará. This program is shared by various associations, forming a network of partnership among breeders, universities and research institutions, such as the Brazilian Agricultural Research Corporation (EMBRAPA) and the Animal Germplasm Bank (BAGAM). The development of research projects aimed to characterize the phenotypic, productive, reproductive, genetic and cytogenetic traits of the horses by Egito et al., (2002).

According Marques et al., (2003) and Costa et al (2005) among breeds present in Brazil the Marajoara breed, with its current contingent of approximately 150.000 individuals, stands out as being extremely well-adapted to the climatic conditions and specific vegetation of Marajó Island (Pará/Brazil). In the island they are fed on rustic fodder and exposed to intense periods of flooding followed by drought, as well as Amazonian diseases. Furthermore, due to these characteristics, the Marajoara horse has been crossed with other naturalized Brazilian breeds losing characteristics as it is interbred.

In terms of cytogenetics, the domestic horse (E. caballus) has $2 \mathrm{n}=64$ chromosomes and was described correctly for the first time by Rothfels et al (1959). This was followed by countless studies on various breeds of the specie aiming the chromosomal characterization and establishing of $\mathrm{C}, \mathrm{G}$ and NOR band patterns by Buckland et al., (1976), Kopp et al., (1981), Lui et al (1990), Richer et al., (1990) and Slota et al., (2007). However, studies about the karyotypic of Marajoara breed are unknown. Therefore, the aim of this study was the cytogenetic characterization of Marajoara horses in terms of diploid number, chromosomal biometry and C- and NOR-banding patterns, as part of the Brazilian genetic resources conservation program.

\section{Methods}

\section{Selection Samples}

The herd of Marajoara horses in the genetic conservation program has 47 animals maintained in the Marajó Island (Pará, Brazil). In this study were selected eight representative specimens (six males and two females) considered the founders of the herd and candidates for reproduction in the program. From each specimen was taken ten milliliters of peripheral blood in vacuum tubes with heparin, kept at $4{ }^{\circ} \mathrm{C}$, and transported from the Marajó Island to the Biology Laboratory of UNIPAMPA (72 hours to culture of samples).

\section{Lymphocyte Culturing}

Metaphases were obtained from lymphocyte cultures according to Moorhead et al., (1960). The cultures were prepared from approximately $1 \mathrm{ml}$ of peripheral blood in $10 \mathrm{ml}$ of RPMI 1640 culture medium, supplemented with $20 \%$ bovine fetal serum, penicillin, streptomycin and phytohemagglutinin. The cultures were incubated at $37^{\circ} \mathrm{C}$ for 72 hours. One hour before the incubation ending, cells were treated with $0.1 \mathrm{ml}$ colchicine solution $(0.05 \%)$ to inhibit cell division in the metaphase stage. Cultures were then hypotonized with $10 \mathrm{ml}$ KCL $0.075 \mathrm{M}$ for 20 minutes and fixed in a solution of methanol and acetic acid (3:1).

\section{Chromosomal Analysis}

Determination of chromosomal number was done through slides in conventional staining ( $1 \mathrm{ml}$ of Giemsa solution for the $9 \mathrm{ml}$ phosphate buffer); it was observed 40 metaphases of each specimen. The representative metaphases were selected and photographed to assemble the individual karyotype following standards for domestic horses, according to Richer et al., (1990). 
In addition, it was estimated the centromeric index CI (ration between short arm and total length of the chromosome), using the software Micro Measure (version 3.3 by Colorado State University) from five different animals karyotypes. The CI parameter was used to define the chromosomal morphology according to Guerra (1986). The determination of the $C$ banding from all chromosomal preparations was done through the protocol of Sumner (1972) and the activity of regions organizers of nucleolus, NORbanding, according to Howell and Black (1980).

\section{Results}

The Marajoara horses showed 2n=64 and its karyotype is representative of a male with XY sex chromosomes, females diverged only by presenting $\mathrm{XX}$ sex chromosomes (Figure 1). The karyotype is split into two main groups, biarmed chromosomes on rows 1, 2 and 3, including the $\mathrm{XX}$ or $\mathrm{XY}$ sex chromosomes, and the remaining single-armed chromosomes on rows 4,5 and 6 .

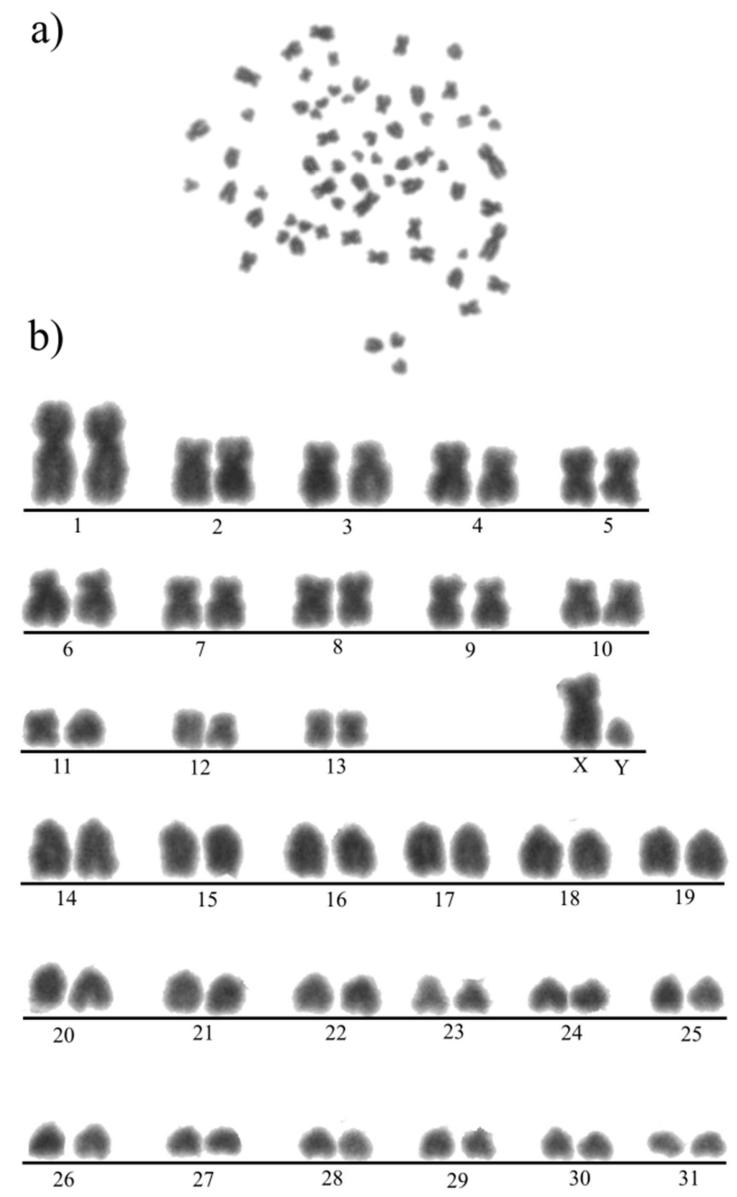

Figure 1: Metaphase and Karyotype of a Male Specimen of the Marajoara Horse, Equus Caballus, 2n=64. A) in the Metaphase, the Arrows Indicate the X and Y Sex Chromosomes; B) the Karyotype is Split into Two Main Groups and Numbered Sequentially. Two-Armed Chromosomes are Grouped Together on Rows 1, 2 and 3 (Chromosome Pairs 1 To 13). The XY Chromosomes are on Row 3. The Remaining Single-Armed Chromosomes are on Rows 4, 5 and 6 (Pairs 14 To 31). 
The averages of the centromeric index (CI) and the morphology of each chromosome pair indicate that horse karyotype consists mainly of submetacentric and telocentric chromosomes, with metacentric morphology only in the $12^{\text {th }}$ pair and no acrocentric morphology in the chromosomal complement (Table 1).

Table 1: Centromeric Index (CI) and Morphology of the Chromosome Complement of Marajoara Horses (Equus Caballus).

\begin{tabular}{|l|l|l|}
\hline Chromosome pair & CI $^{\text {a }}$ & Morphology \\
\hline $\mathbf{1}$ & 0.36 & Submetacentric \\
\hline $\mathbf{2}$ & 0.28 & Submetacentric \\
\hline $\mathbf{3}$ & 0.34 & Submetacentric \\
\hline $\mathbf{4}$ & 0.28 & Submetacentric \\
\hline $\mathbf{5}$ & 0.39 & Submetacentric \\
\hline $\mathbf{6}$ & 0.30 & Submetacentric \\
\hline $\mathbf{7}$ & 0.39 & Submetacentric \\
\hline $\mathbf{8}$ & 0.32 & Submetacentric \\
\hline $\mathbf{9}$ & 0.34 & Submetacentric \\
\hline $\mathbf{1 0}$ & 0.31 & Submetacentric \\
\hline $\mathbf{1 1}$ & 0.39 & Submetacentric \\
\hline $\mathbf{1 2}$ & 0.40 & Metacentric \\
\hline $\mathbf{1 3}$ & 0.35 & Submetacentric \\
\hline $\mathbf{1 4}$ & 0 & Telocentric \\
\hline $\mathbf{1 5}$ & 0 & Telocentric \\
\hline $\mathbf{1 6}$ & 0 & Telocentric \\
\hline $\mathbf{1 7}$ & 0 & Telocentric \\
\hline $\mathbf{1 8}$ & 0 & Telocentric \\
\hline $\mathbf{1 9}$ & 0 & Telocentric \\
\hline $\mathbf{2 0}$ & 0 & Telocentric \\
\hline $\mathbf{2 1}$ & 0 & Telocentric \\
\hline $\mathbf{2 2}$ & 0 & Telocentric \\
\hline $\mathbf{2 3}$ & 0 & Telocentric \\
\hline $\mathbf{2 4}$ & 0 & Telocentric \\
\hline $\mathbf{2 5}$ & 0 & Telocentric \\
\hline $\mathbf{2 6}$ & 0 & Telocentric \\
\hline $\mathbf{2 7}$ & 0 & Telocentric \\
\hline $\mathbf{2 8}$ & 0 & Telocentric \\
\hline $\mathbf{2 9}$ & 0 & Telocentric \\
\hline $\mathbf{3 0}$ & 0 & Telocentric \\
\hline $\mathbf{3 1}$ & 0 & Telocentric \\
\hline $\mathbf{X}$ & 0.30 & Submetacentric \\
\hline $\mathbf{Y}$ & 0 & Telocentric \\
\hline
\end{tabular}

a) Biometric average of five karyotypes of different exemplary, ${ }^{\mathrm{b}}$ morphology defined according to the method proposed by Guerra and Lopes (2002).

The C-banding revealed the presence of constitutive heterochromatin in the centromeric region of all chromosomal complement. For sexual chromosomes it was observed that the $\mathrm{X}$ chromosome exhibited interstitial heterochromatic marking in the short arm while the $\mathrm{Y}$ chromosome is almost entirely heterochromatic and is identified as the smallest telocentric chromosome in the karyotype (Figure 2). 

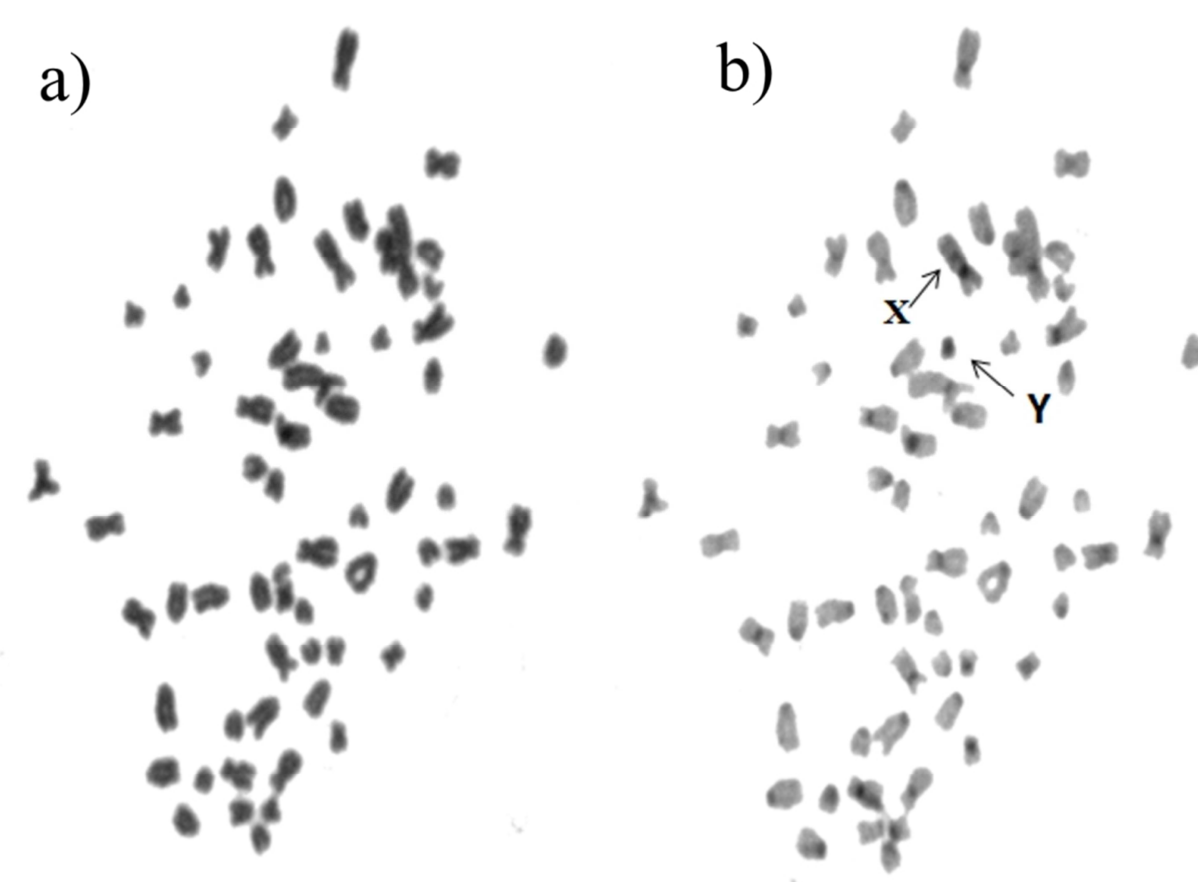

Figure 2: C-Banding Pattern for a Male Specimen of the Marajoara Horse: A) Metaphase with Conventional Staining, B) Banding Showing the Centromeric Markings of the Entire Chromosome Complement (Arrows Indicate Sex Chromosomes: X with Heterochromatic Interstitial Marking on the Q Arm and Y Fully Heterochromatic).

The NOR-banding revealed marking in three chromosome pairs: in the $1^{\text {st }}$ pair in the telomeric region from $\mathrm{p}$ arm, in the $28^{\text {th }}$ pair in the interstitial region near the centromere and in the $31^{\text {st }}$ pair near the centromere (Figure 3, a and b). In addition, we observed expression of homologous pairs in the $1^{\text {st }}$ and in the $28^{\text {th }}$ pairs, and association of the $1^{\text {st }}$ chromosome pair in the nucleolus organization region. 
a)

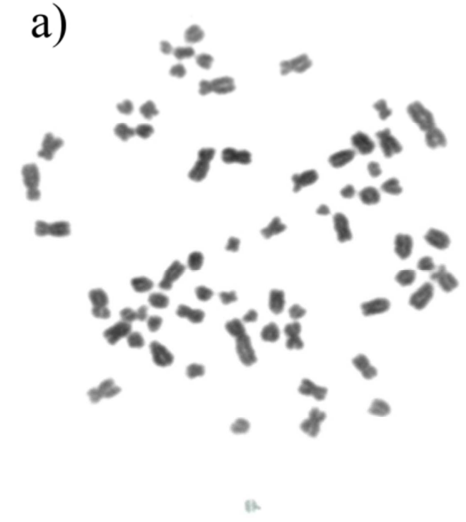

c)

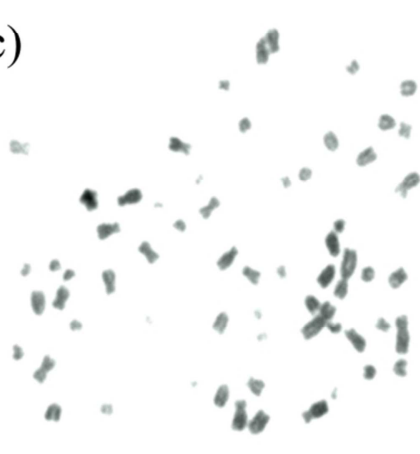

b)

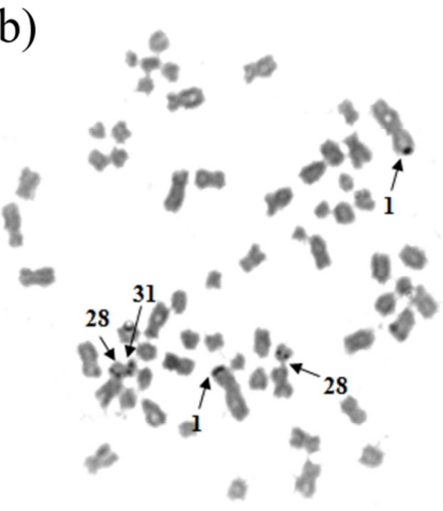

d)

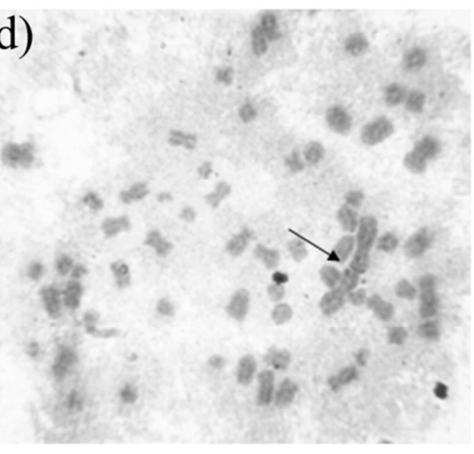

Figure 3: Nucleolar Organizer Regions of a Female Specimen of the Marajoara Horse. A) and C) Show Metaphases in Conventional Giemsa Staining; B) Shows the NOR-Banding with the Telomeric and Interstitial Markings of Both Homologs of Pairs 1 and 28 and the Marking Close to the Centromere on Chromosome 31; D) the Arrow Indicates Association of the $1^{\text {st }}$ Pair in the Nucleolar Organizer Region.

\section{Discussion}

\section{Cytogenetic Characteristics}

This study presents for the first time the karyotype of Marajoara horses. The organization of the chromosomal complement follow the Committee for standardized karyotype of Equus caballus and The Second International Conference for Standardization of Domestic Animal Karyotypes, INRA, Jouy-en Josas, France, 22nd-26th May 1989, published by Richer et al., (1990). However, chromosomal biometry results in this work diverged from the division of karyotype into acrocentric and non-acrocentric groups, as suggested by Richer et al., (1990), since this kind of morphology is not present in the karyotype. In our bibliographical survey, we observed that publications relating to the chromosomal characterization of $E$. caballus did not present a morphological description of each chromosome pair. According to Richer et al., (1990) this may be due to the difficulty of conducting chromosomal biometry to calculate the centromeric index (CI), especially from the smallest chromosomes such as those in the group 26 to 31 .

The C-banding patterns observed in the centromeric region is in agreement Buckland et al., 1976 and are an important parameter to chromosomal biometrics, providing the identification of the centromere in order to define the length of the $\mathrm{p}$ and $\mathrm{q}$ arms for CI calculation, besides the identification of sexual chromosomes.

Regarding the nucleolar organizer regions (NOR-bands), we notice discrepancies in the literature relating to the marking of chromosomes 28 and 31, identified by Kopp et al., (1981) as 25 and 30, by Lui et al., (1990) as 25 and 31 in studies with 
Mangalarga horses. These discrepancies can be due to the polymorphism of the actual length of these small chromosomes, which can vary during different metaphases from one individual to another, making it difficult to identify them correctly on the assembling of the karyotype. The NOR marking in homologous chromosomes identified in our study as pairs 1 and 28 (Figure $3 \mathrm{~b}$ ) were in line with the results of numerous studies and according to Lui et al., (1990) and Slota et al., (2007), this kind of observation can result in different numbers of copies in different lines or rDNA polymorphisms.

The cytogenetical analyses conducted in this study constitute important tools for evaluating animals in terms of the normal diploid number and chromosomal banding patterns for selecting candidates for reproduction free of numeric chromosomal, morphological and structural alterations. This data, together with molecular analyses, can play an important part in identifying the true gene pool for the breed to be conserved.

\section{Marajoara Horses: Historical Aspects and Characteristics}

The horses' importation to Marajó island date from 300 years ago, around 1700, when the first horses were brought from Cabo Verde by Portuguese colonists. The Marajoara horses are originated from crosses between Arab and Anglo-Arab breeds, and over the years have developed its own characteristics and adaptations to the Amazon region. According to Brazilian Association of Horse Breeders of Marajoara - ABCCRM, the breed has characteristics as strength, speed of short gallops, hardiness and versatility as well as adaptation to the flooded areas and rustic foods found on the island. Because of these characteristics, the Marajoara horse has fundamental importance for the development of cattle raising activities, the extensive management of buffaloes and cattle in traction and transportation.

The standards of Marajoara horses breed are a medium sized well proportioned and defined musculature, all peltry patterns except Pampa and Albin, sub-convex profile and hooves rounded and preferably black. Nevertheless, the current phenotypic of Marajoara demonstrate that the horse is in process of mischaracterization, mainly due to indiscriminate breeding that occurred with Quarter Horses, used for the purpose of improving the breed and increase in size. Nowadays, there are not many males and females within the standard features of the original Marajoara horse as the set by ABCCRM, in this context the EMBRAPA have been developed activities to promote the conservation of these animals.

\section{Conservation Program of Marajoara Breed}

The animals kept in the conservation program are the most representative of the phenotype set for race Marajoara; currently the herd program comprised 47 animals. The genetic conservation program is fairly complex in terms of the human and financial resources required; it involves breeders, universities and scientific organizations, which together developed the research to define practical actions for implementation in germplasm banks and farms.

\section{Conclusion}

This was the first cytogenetic study on Marajoara horses, which, in common with other breeds of E. caballus, have a chromosome number of $2 n=64$. The karyotype consists mainly of submetacentric and telocentric chromosomes, but also contains metacentric morphology in the $12^{\text {th }}$ chromosome pair. C-banding revealed a predominance of marking in centromeric regions throughout the entire autosomal complement and is an excellent tool for identifying the $\mathrm{X}$ and $\mathrm{Y}$ sex chromosomes. The nucleolar organizer regions are located in three chromosome pairs: 1, 28 and 31 Altogether, our study represents one stage in the conservation process and is intended to increase the number of animals evaluated based on various parameters and providing further data of the Marajoara breed. 


\section{Acknowledgments}

Special thanks to the technicians and researchers at Embrapa for sampling the animals, to our colleagues at the laboratory of the Federal University of Pampa who participated in cell culturing and analysis, to the Brazilian National Research Council (CNPq) for funding the study, and to the Academic Development Grants Program from UNIPAMPA (PBDA/UNIPAMPA).

\section{References}

Aaron Reeves, Jim Tear and Colorato State University (1997-2000). Micro Measure version 3.3 (http://www.colostate.edu/Depts/Biology /MicroMeasure/bugs.htm)

Buckland, A. R., Fletcher, J. M. \& Chandeley, A. C. (1976). "Characterization of the Domestic Horse (Equus Caballus Karyotype Using G- and C-Banding Techniques," Experientia, 32 (9) 1146-1149.

Costa, M. R., Marques, J. R. F., Veja-Pla, J. L., Bermejo, J. V. D., Sampaio, M. I. C. \& Gallardo, P. P. R. (2005). "Variabilidade Genética de Equinos da Amazônia Brasileira," Ciência Biotecnologia Desenvolvolvimento, 35 (5) 52-55.

Egito, A. A., Mariante, A. S. \& Albuquerque, M. S. M. (2002). "Programa Brasileiro de Conservação de Recursos Genéticos Animais," Archivos de Zootecnia, 51 (194) 39-52.

Guerra, M.S. (1986). 'Reviewing the Chromosome Nomenclature of Levan et al,' Rev Revista Brasileira de Genetica, 9 (4) 741-743.

Howell, W. M. \& Black, D. A. (1980). "Controlled Silver Staining of Nucleolus Organizer Regions with a Protective Colloidal Developer: A L-Step Method," Experientia, 36 (8) 1014-1015.

Kopp, E., Mayr, B., Czaker, R. \& Schleger, W. (1981). "Nucleolus Organizer Regions in the Chromosomes of the Domestic Horse," Journal of Heredity, 72 (5) 357-358.
Lui, J. F., Giannoni, M. A., Giannoni, M. L. \& Tosta, P. A. (1990). "Characteristics of NorBanded Chromosomes of Mangalarga Horses," Brazil Journal of Genetics, 13 (2) 269-281.

Mariante, A. S. \& Egito, A. A. (2002). "Animal Genetic Resources in Brazil: Result of Five Centuries of Natural Selection," Theriogenology, 57 (1) 223-35.

Marques, J. R. F., Costa, M. R., Egito, A. A., Mariante, A. S. \& Albuquerque, M. S. M. (2003). "Conservation of Genetic Resources of the Small Populations of Domestic Animal of the Amazon Region in Brazil," Animal Genetic Resources Information, (33) 31-40.

Moorhead, P. S., Nowell, P. C., Mellinan, W. J., Battips, D. M. E. \& Hungerford, D. A. (1960). "Chromossome Preparations of Leukocytes Cultured from Human Peripheral Blood," Experimental Cell Research, 20 (3) 613-616.

Richer, C. L., Power, M. M., Klunder, L. R., Mcfeely, R. A. \& Kent, M. G. (1990). "Standard Karyotype of the Domestic Horse (Equus Caballus)," Hereditas 112 (3) 289293.

Rothfels, K. H., Axelard, A. A., Siminovitch, L., Mcculloch, E. A. \& Parker, R. C. (1959). 'The Origin of Altered Cell Lines from Mouse, Monkey and Man as Indicated by Chromosome and Transplantation Studies,' In III Canadian Cancer Conference Academic Press Inc New York (2007), 189-214.

Slota, E., Wnuk, M., Bugno, M., PienkowskaSchelling, A., Schelling, C., Bratus, A. \& Kotylak, Z. (2007). "The Mechanisms Determining the Nucleolar-Organizing Regions Inactivation of Domestic Horse Chromosomes," Journal of Animal Breeding and Genetics, 124 (3) 163-171.

Sumner, A. T. (1972). "A Simple Technique for Demonstrating Centromeric Heterochromatin," Experimental Cell Research, 75 (1) 304-306. 